\title{
Application of Multipoint Electrodes to Telemetry in Patient-monitoring and During Physical Exercise
}

\author{
DAVID LEWES AND D. W. HILL \\ From the Cardiac Unit, Bedford General Hospital, and the Research Department of Ancesthetics, \\ The Royal College of Surgeons, London W.C.1
}

Telemetry (Gk: Tédos, end; $\mu \epsilon ́ \tau \rho o v$, measure) is defined as the making of measurements at a distance from the subject.

Following the successful use of a dry multipoint electrode for routine electrocardiography (Lewes, 1965, 1966; Stock, 1966; Turner, 1966; Keen and Johnson, 1966), we have conducted preliminary trials using a multipoint chest electrode for the monitoring by radio-telemetry of patients with myocardial infarction and in the assessment of cardiac function during physical exercise. Latterly our studies have embraced the transmission of the electrocardiogram over the General Post Office (G.P.O.). telephone system using multipoint electrodes and transistorized electrocardiographs.

Resuscitation in the intensive care units in the United Kingdom involves monitoring by conventional methods which include the wiring of complex apparatus at the bedside directly to the patient. This state of affairs is far from ideal since modern electronic equipment, especially when incorporating an oscilloscope and alarm devices, aggravates the emotional tension and anxiety of a seriously ill patient already predisposed by cardiac infarction to dangerous dysrhythmias. Indeed, it is likely that such monitoring equipment, designed to anticipate and detect dysrhythmia, occasionally precipitates the disorder it is meant to prevent, especially in the seriously ill and emotionally disturbed subject.

Current methods of bedside monitoring suffer the added disadvantage that, in the event of an emergency necessitating external cardiac massage, wires connecting patient and adjacent apparatus are an encumbrance to resuscitation procedures. From the patient's point of view, monitoring by telemetry is ideal since it overcomes these disadvantages. Radiotelemetry, moreover, is obligatory for the electrocardiographic assessment of the cardiac state under

Received September 23, 1966.

4 conditions where conventional electrocardiography is either impractical or impossible, for example, during a conventional exercise test or while running.

Since a multipoint electrode requires no preparation with jelly or paste and achieves skin contact at numerous points by penetrating the stratum corneum, which is responsible for the greater part of natural skin resistance (Robertson, 1937), artefact skin potentials due to electrode-skin movement may be greatly diminished (Fig. 1). Movement of a flat metal electrode, separated from the skin by a fluidconducting medium, produces electrical potentials at the electrode/jelly/skin interfaces which are reflected in the radiocardiogram or conventional electrocardiogram by artefact potentials, or as a wandering baseline (Boter, den Hertog, and Kuiper, 1966).

\section{ApPaRATUS AND METHODS}

Radio-telemetry Systems. Crystal-controlled transmitters and receivers were used throughout (Fig. 2). The term "crystal-controlled" means that reception by the receiver is locked to the transmission, thus making retuning of the receiver unnecessary.

The first system employed was the R.K.G. 100 by Telemedics of Southampton, Pennsylvania. It is a frequency-modulated system, having a carrier frequency of 80 megacycles per second and a range of 100 yards. The second system was the C \& M Radiocardiograph produced by Medical \& Industrial Equipment Ltd., of London. It is an amplified and frequency-modulated system operating at $169 \mathrm{Mc} / \mathrm{s}$. AM/FM uses a combination of amplitude and frequency modulation: amplitude modulation alters the amplitude of the transmitted carrier wave, and frequency modulation alters the frequency of the carrier. Both telemetry systems can be adapted to operate in the $102 \cdot 2-102 \cdot 4 \mathrm{Mc} / \mathrm{s}$ band proposed for medical radio-telemetry by the G.P.O. A G.P.O. licensing fee of 20 shillings is necessary for users of medical radio-telemetry equipment and covers a fiveyear period.

Most of our radio-telemetric recordings were made 


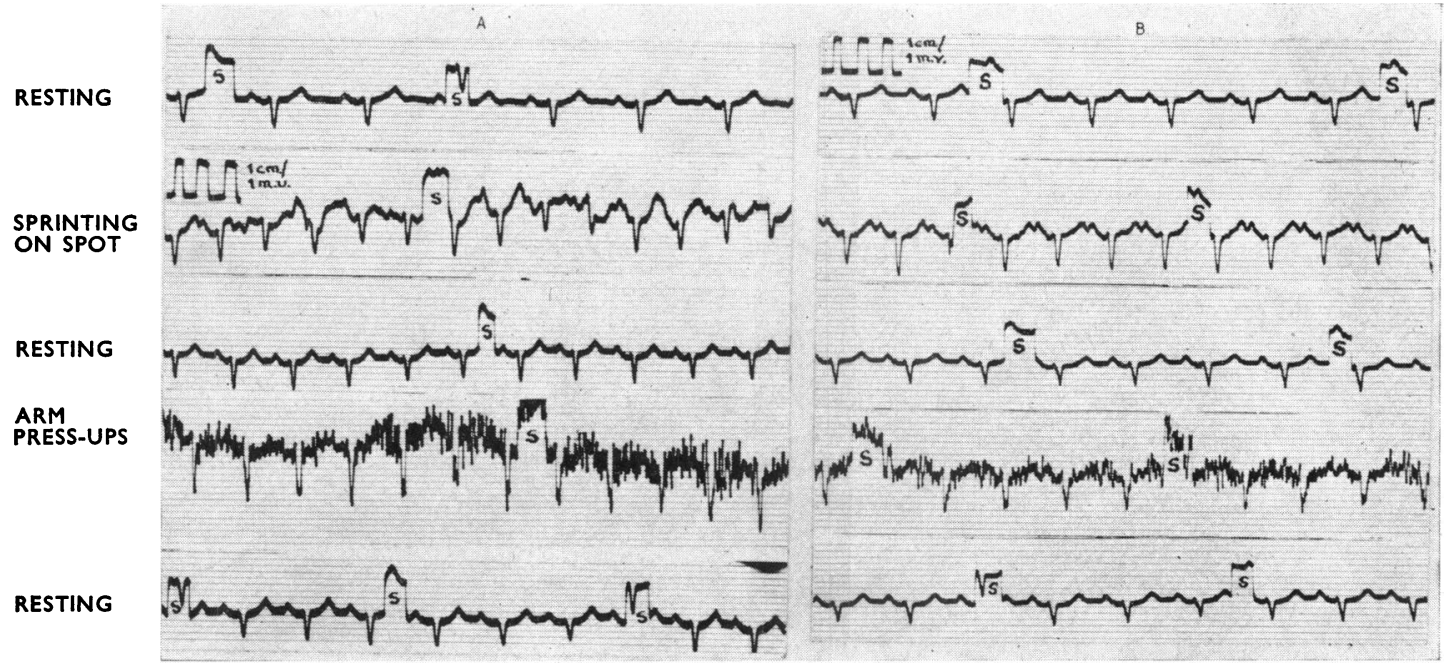

Frg. 1.-Electrocardiograms recorded in a healthy man of 50, using in (A) two standard Philips suction cloth electrodes, and in (B) two multipoint suction electrodes, applied to the manubrium sterni and xiphisternum. Note the steady baseline and reduction of artefact potentials during exercise in (B).

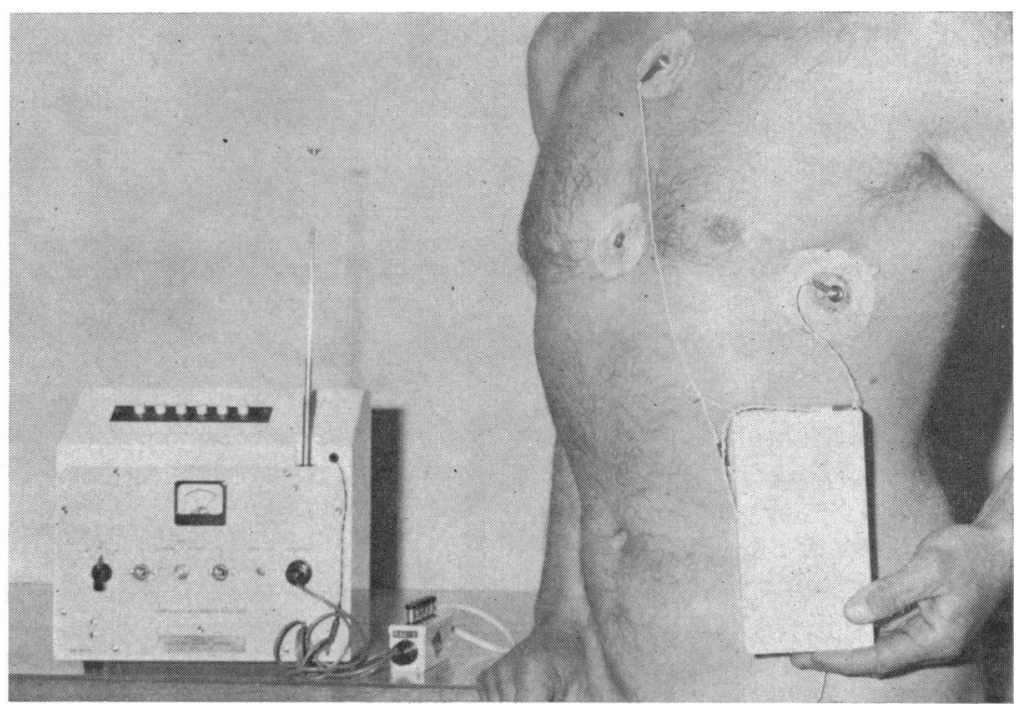

FIG. 2.-The C \& M Radiocardiograph (Medical \& Industrial Equipment Ltd.), showing the transmitter input connected to the chest electrodes and receiver with output terminal block for the recording apparatus.

with the British telemetry system which has been available in the country for more than five years at a total cost less than that of a reliable modern single-channel electrocardiograph. The transmitter and receiver are each powered by a compact standard radio 9-volt battery (Ever Ready PP6 or equivalent) which operates with intermittent use for $40-50$ hours and continuously for approximately 10 hours; renewal of batteries is inexpensive, simple, and quickly effected.

The transmitter and receiver, each with its self-con- tained aerial and FM/AM circuits, normally reject all unwanted radio signals, so that only electrical signals from the patient are transmitted and received by the telemetry equipment. The transmitter and receiver, including batteries, weigh approximately $566 \mathrm{~g}$. and $2.26 \mathrm{~kg}$. (20 oz. and $5 \mathrm{lb}$.), respectively.

The recorders used with these systems were: Philips Cardiopan 1, Elmqvist Junior portable, Newmark Cardette (all single-channel), and the Mingograf-24B (double channel). 
Electrodes. Circular multipoint electrodes of $2 \cdot 8 \mathrm{~cm}$. diameter, made from a tin-plated soft iron nutmeg grater (Lewes, 1965), were soldered to a centrally placed pressstud contact. A centrally perforated disc of thick paper $2.8 \mathrm{~cm}$. diameter was glued to the back surface of each electrode. A disc of coarse linen approximately twice the diameter of the electrode was lightly attached to the electrode, which was then steam-sterilized.

Electrode Application. The most suitable electrodeskin areas, in regard to the production of minimal electromyographic activity, have been found to be over the manubrium sterni and the fifth or sixth left intercostal space just anterior to the mid-axillary line. The selected areas were shaved, if necessary, and thoroughly cleaned with ether. The sterilized electrode was pressed firmly into position on to the cleaned, stretched skin. The linen disc, previously saturated on one side with a non-irritant waterproof glue ${ }^{\star}$, was then affixed to the electrode and surrounding skin, which was well stretched with the fingers of one hand during application. Firm pressure was maintained for several minutes until the glue had set; this process was hastened by using a hair dryer, as first suggested by a patient. An application of the glue to the outer surface of the linen disc ensured a waterproof arrangement which was unaffected by daily bathing. Unless the electrode be firmly applied and securely glued in position, a varying skin-contact resistance due to electrode movement may mar the radiocardiogram during physical activity. Skin-contact resistance is also altered by direct pressure on the electrode.

The Telemedics RKG 100 receiver contains a skin resistance meter, and with proper electrode application the skin contact resistance for paired electrodes was usually less than $10 \mathrm{k} \Omega$ at 20 c.p.s., but readings as low as $3 \mathrm{k} \Omega$ were occasionally observed. An experimental latex gluet is at present being evaluated and has given promising results.

The transthoracic lead gives a tall $R$ wave and positive $T$ wave in normal subjects and proved satisfactory both for patient-monitoring and for interpretation of the electrocardiogram during physical exercise.

\section{MONITORING BY RADIO-TELEMETRY IN MYOCARDIAL INFARCTION}

Small diameter patient leads were connected to the studs of the electrodes and to the input terminals of the transmitter which was placed in the patient's pyjama pocket, or secured beneath his pillow. The receiver was located in a central monitoring area, its output being fed to a conventional electrocardiograph and oscilloscope to produce signals identical with those normally recorded at the bedside (Fig. 3).

Results. Preliminary studies in four subjects have shown that satisfactory monitoring by radio-telemetry using multipoint electrodes can be achieved

* Type Saba-select FH by Depex Ltd., Debilt, Holland.

† Manufactured by I.C.I. Pharmaceuticals Ltd. with a minimum of mental and physical disturbance to the patient, not only in acute cardiac infarction when a patient might be desperately ill and apprehensive (Fig. 4), but also for periods of up to two weeks during convalescence (Fig. 3). There was no complaint of discomfort from the electrodes which, in two patients, were worn continuously while undertaking normal convalescent activities. Skin damage, moreover, was negligible, the superficial abrasion requiring no dressing after removal of the electrodes. In no patient has infection or skin reaction due to the glue or metal been observed following the application of the sterilized multipoint electrodes.

\section{RADIO-ELECTROCARDIOGRAPHY DURINg Physical EXercise}

The use of radio-telemetry techniques to record electrocardiograms from exercising subjects is by now well known (Bellet et al., 1962). It might be expected, however, that the use of dry multipoint electrodes would reduce motion artefact signals. Employing the technique of electrode application described above, radiocardiograms both at rest and during physical exercise were made during a 16week period on a man aged 50 years in whom the left axillary multipoint electrode remained in its original position throughout this time (Fig. 5). After 18 days the manubrial electrode stud attachment became dislodged, necessitating renewal of the entire electrode. The resultant skin damage was slight and consisted of punctate areas of epithelial hyperplasia roughly corresponding to the points of the electrode, as well as light pigmentation due to chronic irritation (Fig. 6).

The original left axillary electrode, in position more than four months later, required further application of glue to the cloth disc which eventually became rigid necessitating renewal every few weeks; this change was made without disturbing the attachment of the multipoint electrode to the chest wall.

For four months our subject led a full and active life, took a daily bath, and was undisturbed by the presence of the electrode. Although physically fit he happened to be liable to attacks of paroxysmal atrial fibrillation customarily suppressed by digoxin $0.75 \mathrm{mg}$. daily. While in sinus rhythm there were no abnormal cardiac symptoms, even on strenuous exertion, but when the fibrillation set in, effort dyspnoea and palpitation were troublesome. Normal rhythm was promptly restored by propranolol $20 \mathrm{mg}$. orally (Fig. 7).

Results. During strenuous physical exercise, such as running up and down stairs, multipoint chest 


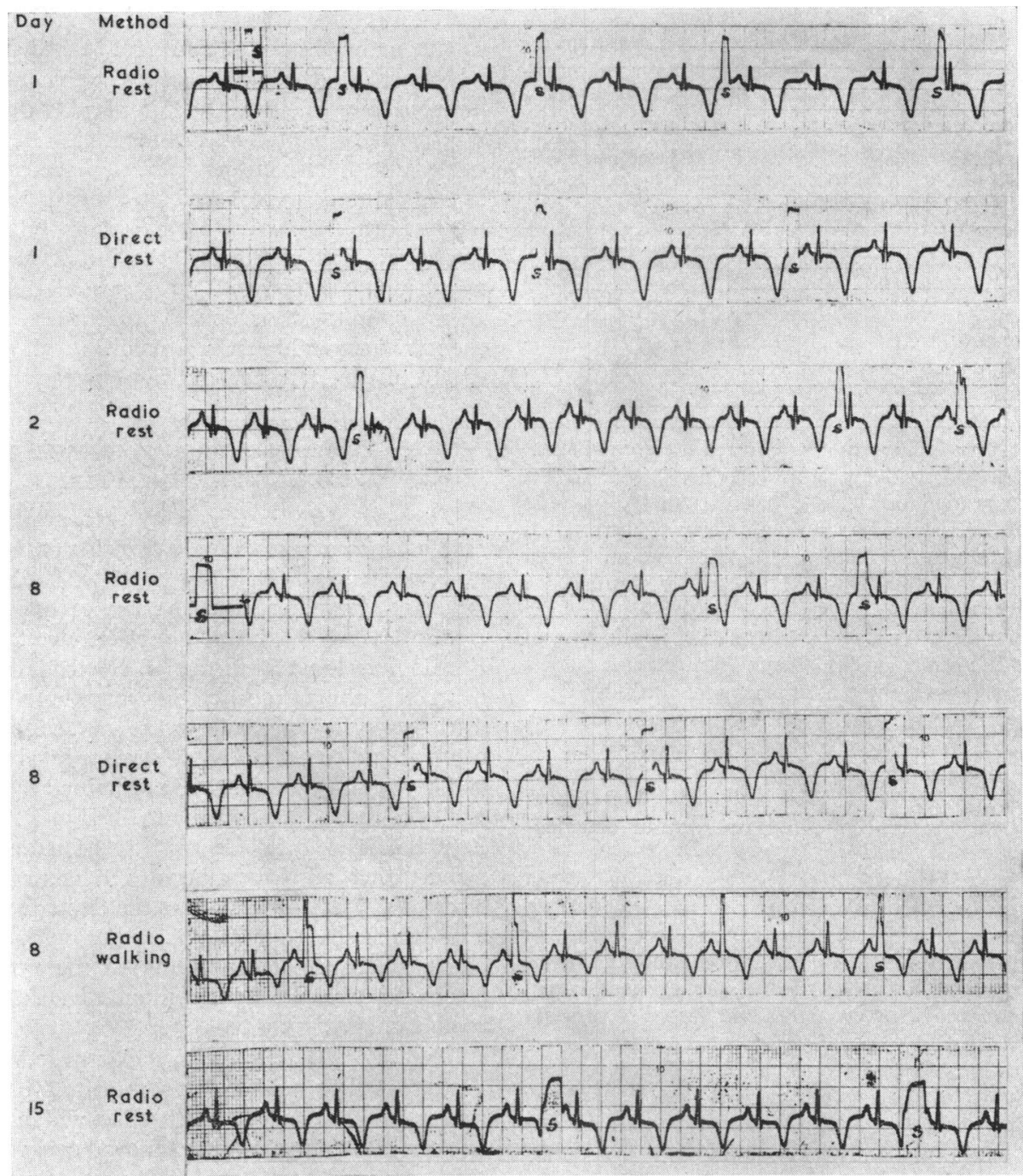

FIG. 3.-Serial radiocardiograms and direct electrocardiograms recorded in a man of 54 over a 2-week period during convalescence following postero-lateral cardiac infarction, using multipoint chest electrodes applied to the manubrium sterni and left axilla (see text).

electrodes, when correctly applied, yielded a radiocardiogram with a consistently steady baseline and minimal artefact potentials, factors that may be undesirable as they render interpretation difficult or impossible when using conventional electrodes and abrasive saline jelly (Fig. 7 and 8 ).

Throughout the 16-week period of observation radiocardiograms have been recorded on 11 different occasions at intervals of a week or more during such activities as running on the level or upstairs, step tests, or sprinting on the spot. In each of five such examinations, controlled by conventional electrodes and abrasive saline jelly, the continuous tracings obtained with the dry multipoint electrodes were unequivocally superior to the control records (Fig. 8). The final radiocardiogram was recorded during a step-test 116 days after the original application of the left axillary electrode (Fig. 9). The contact resistance for the paired chest electrodes had now risen to $20 \mathrm{k} \Omega$ (d.c.), and it was not possible to record an acceptable radiocardiogram during more vigorous exercise.

The left axillary electrode, when removed four days later, was rusted and plugged with epithelial 


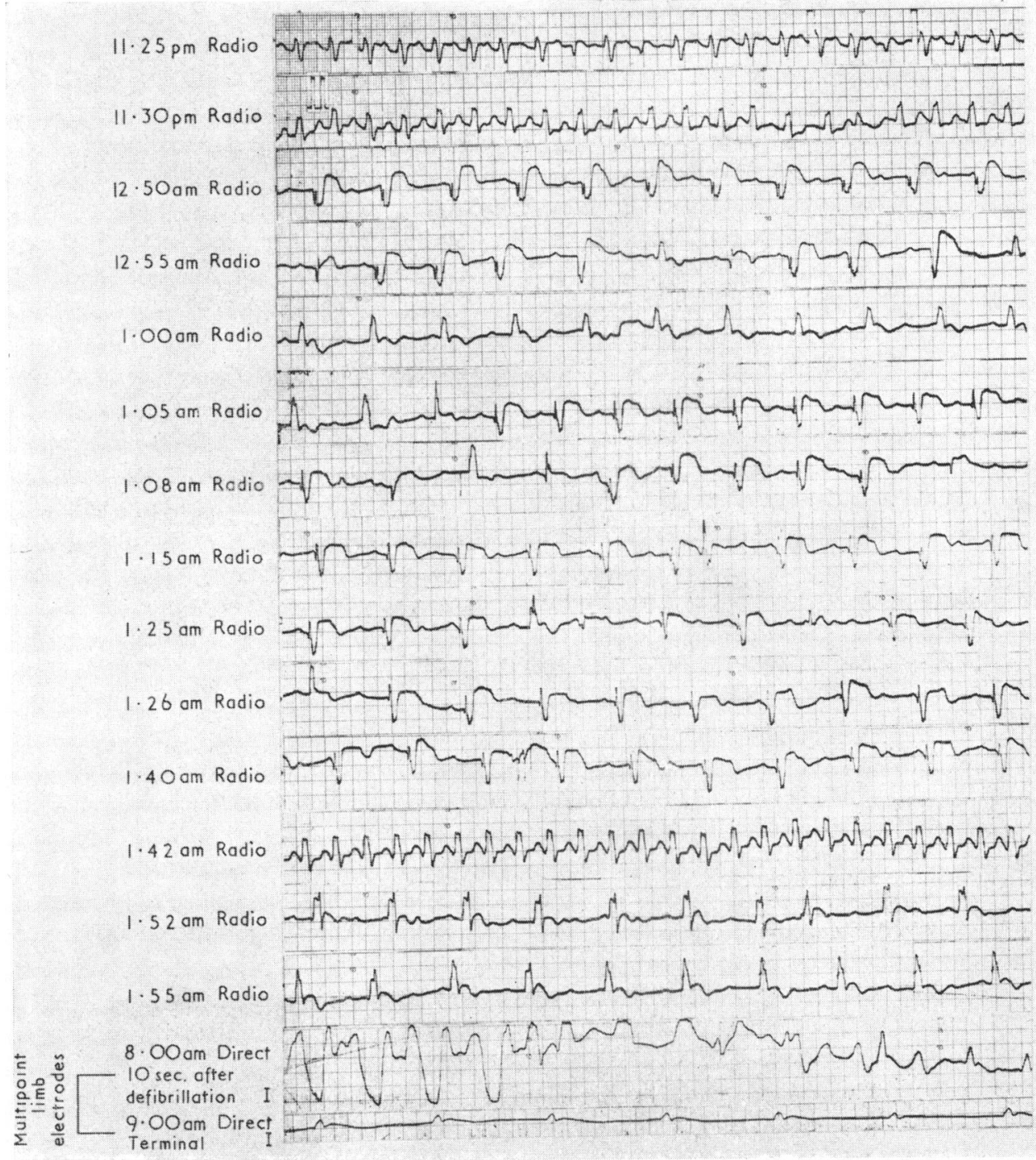

FIG. 4.-Serial radiocardiograms recorded during a $9 \frac{1}{2}$-hour period in a man of 57 dying within 24 hours of massive anterior cardiac infarction, confirmed at necropsy. Multipoint electrodes were applied to the manubrium sterni and mid-axillary line in the sixth intercostal space.

debris; the underlying skin, however, was smooth and unbroken, showing only punctate areas of dark pigmentation.

\section{TRANSMISSION OF ELECTROCARDIOGRAMS BY G.P.O. TELEPHONE LINES}

The first known demonstration in Great Britain of the transmission of an electrocardiogram and other biological waveforms by G.P.O. telephone lines was given on March 29, 1966, at the Biomedical Exhibition at the New Horticultural Hall, Westminster, by Professor J. P. Payne and one of us
(Hill, 1966), with the co-operation of G.P.O. engineers.

Method and Apparatus. A standard Post Office Datel Modem equipment was modified to provide an F.M. system with a carrier frequency of 1700 c.p.s. The electrocardiogram was recorded with a Cardette transistorized electrocardiograph by Louis Newmark Ltd., which had been fitted with a transistorized single-sided output stage to feed the Datel Modem. The output from the Modem was fed to an ordinary telephone line obtained by normal dialling. At the receiving end another Modem fed a second Cardette. The patient was fitted with a set of multipoint limb electrodes. 


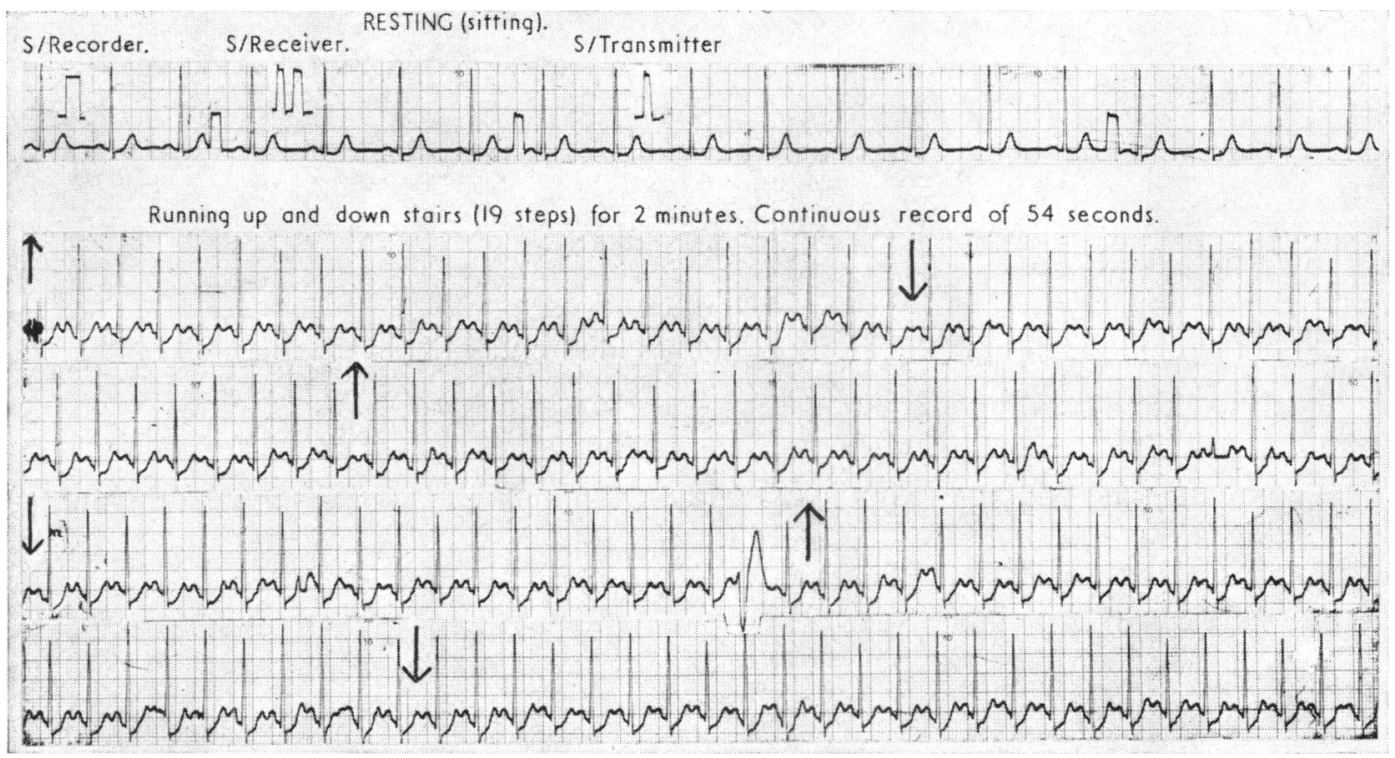

FIG. 5.-Radiocardiogram at rest and during physical exercise in a man of 50 years. Multipoint chest electrodes applied to the xiphisternum and left mid-axillary line 114 hours previously and worn continuously during normal daily activities. Skin contact resistance of paired electrodes $7 \mathrm{k} \Omega$ (a.c.) before and after recording.

The patient was a 44-year-old man undergoing a urinary bladder operation at St. Paul's Hospital, London. The electrocardiogram and pneumotachogram were displayed on 7 a Cardette electrocardiograph and a pair of 17-inch display cathode ray tube oscilloscopes at the New Horticultural Hall two miles from the hospital. One millivolt calibration pulses and the standard limb leads were transmitted. There was no significant degree of interference present on the tracings (Fig. 10).

\section{Discussion}

Multipoint electrocardiography without skin preparation is receiving increasing support as a

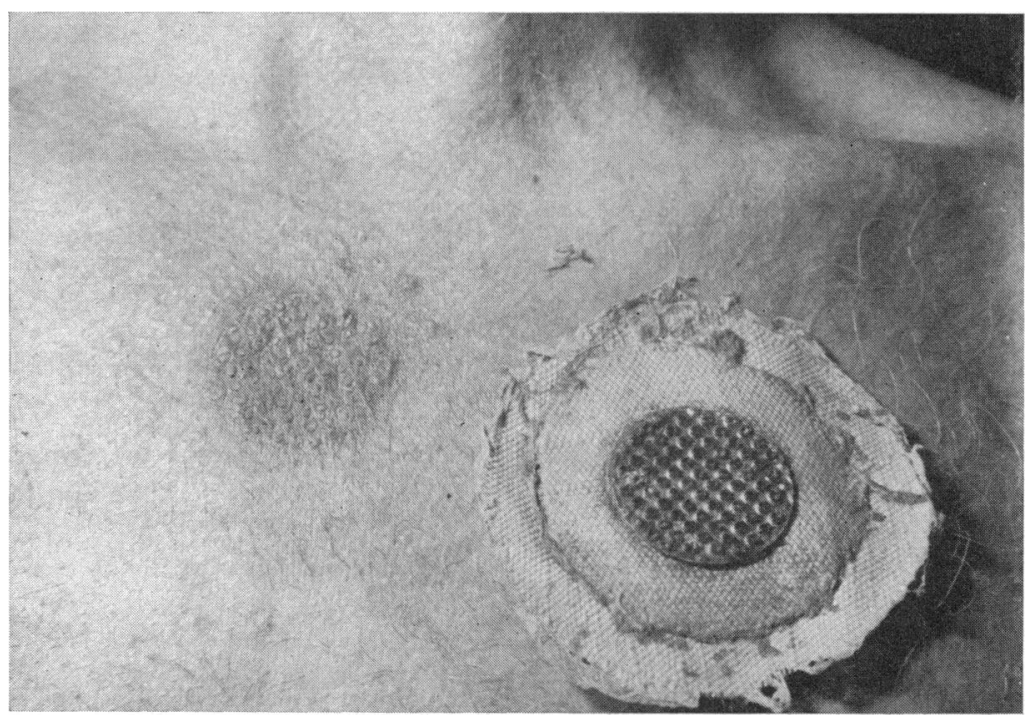

FIG. 6.-Multipoint chest electrode and electrode skin area after 18 days of continuous application. Note the punctate areas of epithelial hyperplasia and darkening of the skin due to local erythema and pigmentation. 


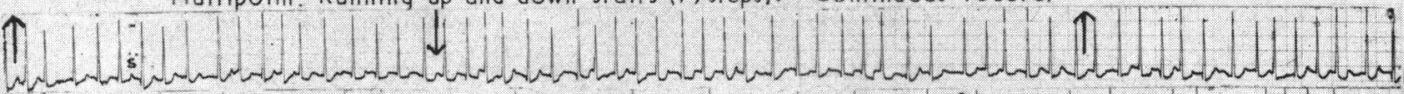

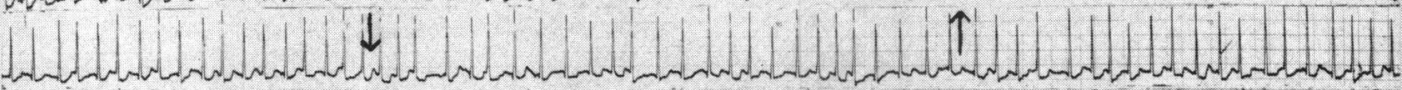

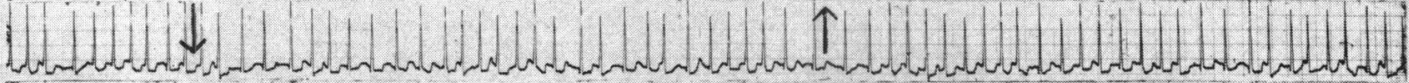

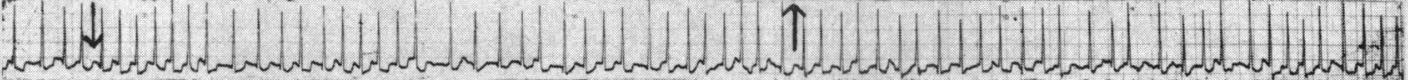

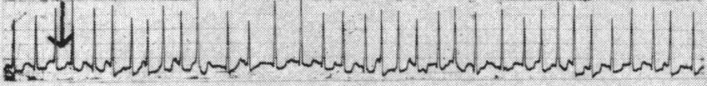

6 hours later. Normal rhythm Multipoint: Rest.

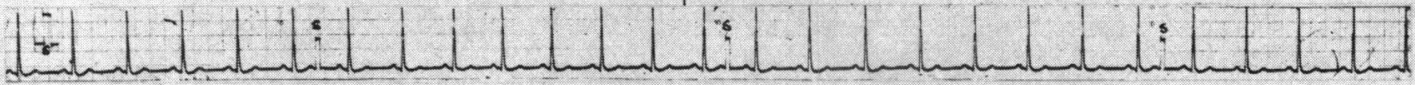

Multipoint: Running up and down stairs (19 steps). Continuous record

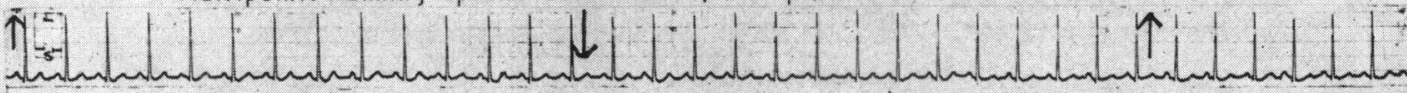

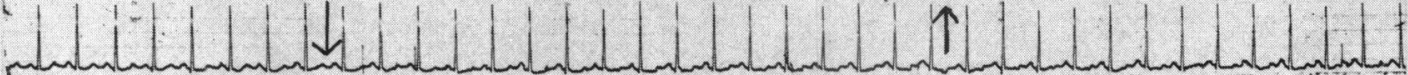

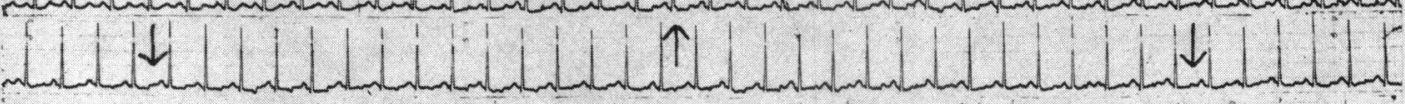

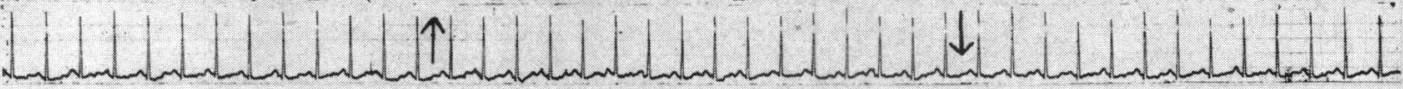

Control: Rest $C \& M$ electrodes and Cambride Jelly.

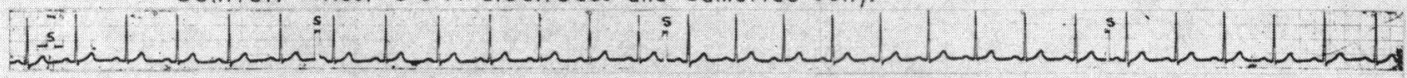

Control: Running up and down stairs (19 steps)-Cambridge Jelly.

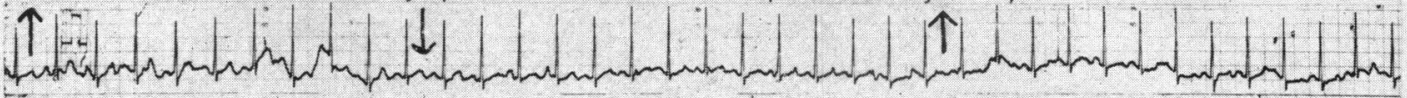

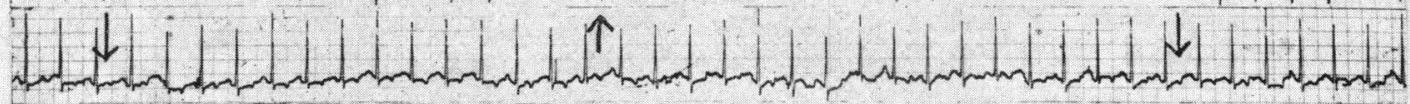

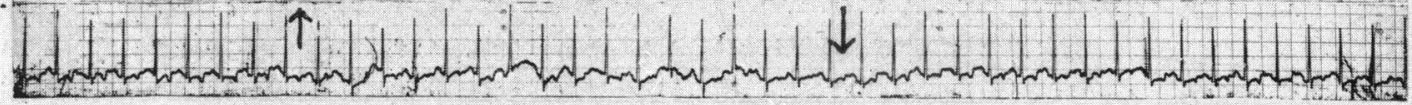

FIG. 7.-Continuous radio-telemetric records at rest and during exercise using multipoint chest electrodes in a man of 50, in whom the left axillary electrode had been continuously in position for 36 days. Top records: atrial fibrillation. Middle records: normal rhythm after propranolol. Bottom records: control tracings in normal rhythm using conventional electrodes and Cambridge jelly. 


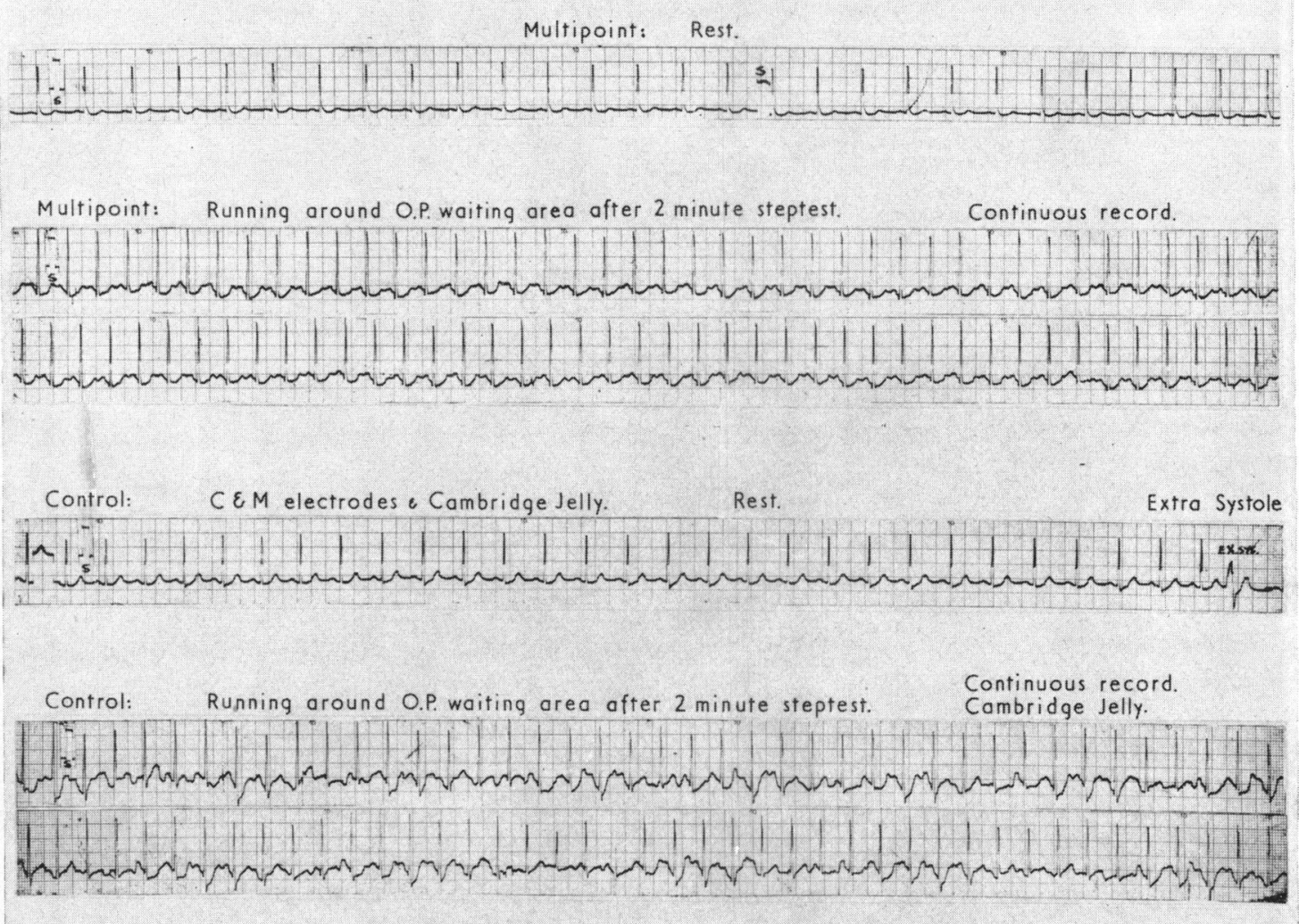

FIG. 8.-Continuous radio-telemetric records during exercise, using multipoint electrodes and control tracings employing conventional electrodes and Cambridge jelly. The left axillary multipoint electrode had been continuously in position for 25 days.

convenient, clean, and time-saving method of recording electrocardiograms from the body surface (Stock, 1966; Turner, 1966; Wilton-Davies, 1966; Ernsting, 1966; Keen and Johnson, 1966).
For routine electrocardiography and patient monitoring, and for survey work when saving time is important, the method has been found quick and reliable, yielding records indistinguishable from

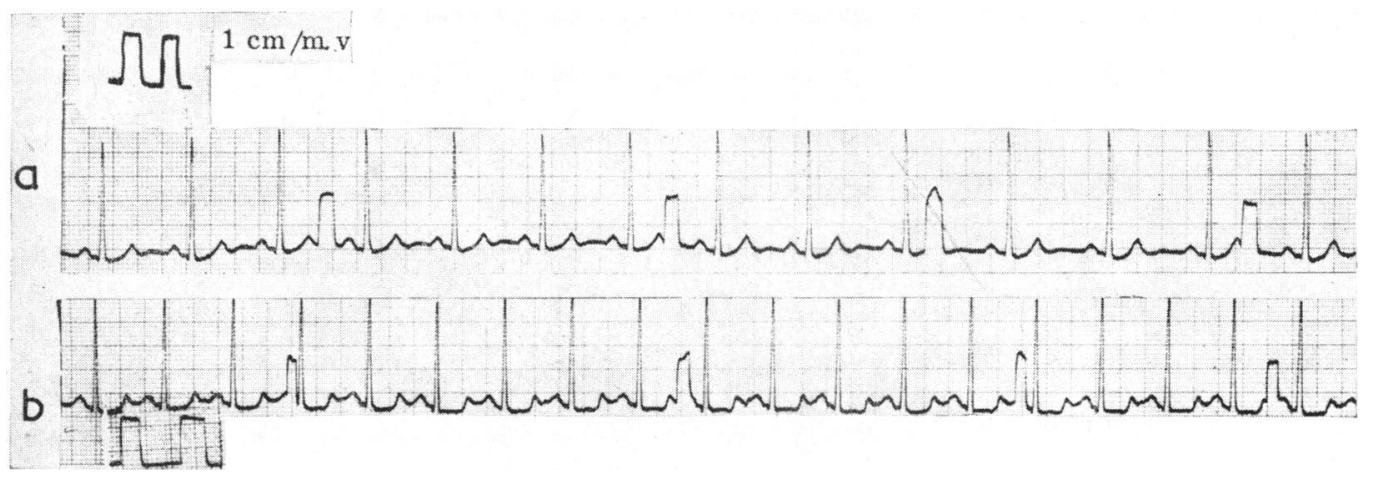

FIG. 9.-Radiocardiogram (a) at rest and (b) during a step-test, using multipoint electrodes and recorded 116 days after the original application of the left axillary electrode. Contact resistance for paired electrodes $20 \mathrm{k} \Omega$ (d.c.). 


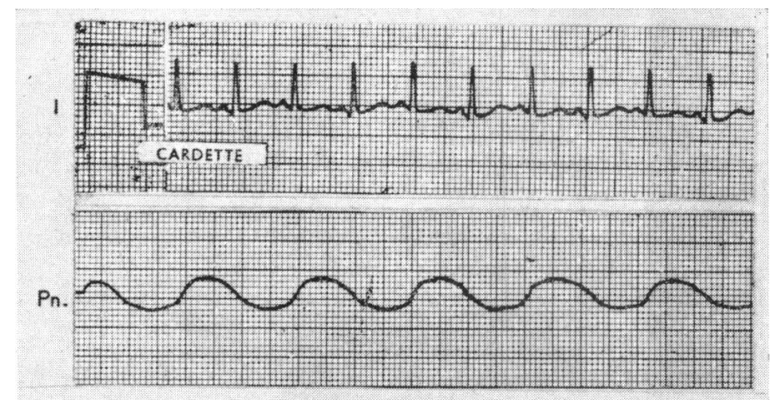

FIG. 10.-Electrocardiogram (I) and pneumotachogram $(\mathrm{Pn})$ of a patient during operation, which were transmitted over the G.P.O. telephone system and recorded on two display cathode ray oscilloscopes and by a transistorized electrocardiograph two miles from the operating theatre. (See text.)

those obtained in the same subjects with conventional electrodes and saline jelly (Stock, 1966; Keen and Johnson, 1966).

Satisfactory electrocardiograms using only multipoint electrodes have been obtained at the high Himalaya (Turner, 1966), in the decompression chamber (Ernsting, 1966), and by telemetry under conditions of increased barometric pressure equivalent to 100 feet of sea water (Wilton-Davies, 1966).

Direct electrocardiograms have been recorded with multipoint electrodes in horses (Lewes, 1965; A. Stevenson, personal communication, 1966). One of us (D. W. H.), working with Professor L. A. Geddes, at the Texas University College of Veterinary Medicine, has recently recorded equine electrocardiograms by radio-telemetry using multipoint electrodes. Coarse stainless steel grater electrodes, of approximately $13 \mathrm{sq} . \mathrm{cm}$. (2 sq. in.), were applied to the unclipped skin of the legs and thorax. The tracings recorded both by direct and radio-telemetric methods were indistinguishable from those obtained after subsequent preparation of the multipoint electrodes and skin with concentrated saline solution. Finally, Wilton-Davies (1966) has recorded oculograms in human subjects with multipoint electrodes, which have proved the equal in performance and the superior in convenience of application to suction cup electrodes and saline jelly.

The present study explores the possibilities of the application of multipoint chest electrodes to radiotelemetry, not only for patient monitoring in cardiac infarction, but also in assessing cardiac function during differing forms of physical activity which preclude the use of conventional electrocardiography. It has been shown that a relatively crude multipoint electrode, efficiently applied, is capable of yielding a high quality radiocardiogram during physical exercise days or weeks after its original application to the chest. Lastly, the value of the dry multipoint electrode in patient-monitoring during surgical operation, and the future possibilities in Great Britain of the transmission of the electrocardiogram and other biological wave forms over the G.P.O. telephone system to distant centres for immediate interpretation or storage by tape for computer analysis, have been indicated.

It is the present intention of the G.P.O. to limit the transmission by radio of physiological signals to a distance of some 600 feet. Patient mobility can be maintained at greater distances by feeding the output of the radio receiver into a Datel Modem and thence to a G.P.O. telephone line, and we have demonstrated this. It may be desirable, however, to monitor a patient having a transient cardiac dysrhythmia over periods such as a normal working day. In this situation it is convenient for the patient to wear a miniature tape recorder which will record the electrocardiogram (Holter, 1961).

In the field of patient-monitoring in cardiac infarction, we consider that telemetry may eventually replace conventional methods. Both clinical observation and the volunteered statements of patients who have survived critical cardiac infarction indicate beyond doubt that frightening electronic equipment and alarm signals at the bedside destroy equanimity, heighten emotional tension, and militate against recovery by rendering a susceptible patient even more liable than his disease dictates to the dangerous dysrhythmias which all too frequently determine an early demise.

Apart from aggravating emotional tension in a seriously ill patient, there are the practical difficulties in the nursing and emergency treatment of a patient wired to numerous instruments at the bedside. G. E. Godber (personal communication, 1966) has stated that: "Telemetry in this field is certainly one of the more important developments. ... One of our troubles in units for acute cardiac infarction is 
the ease with which leads become detached ... and so one does look to this sort of development as soon as possible."

The superior records that may be obtained by multipoint electrodes during physical exercise, compared with those given by conventional jelly electrodes, point the way to the ideal form of multipoint electrode which should be developed in the immediate future. The electrode should be as small as possible yet capable of a low skin-contact resistance by embodying the maximum number of points per unit area, each of which can penetrate the insulating keratinized skin layer. It should require neither jelly nor paste and be dry, apart from the accumulation of natural sweat and electrolytes beneath its surface, so that it may be left in position for days or weeks, a necessity, for example, during prolonged observation in the compression chamber or in astronauts.

Both for long-term patient-monitoring and during exercise studies, it is desirable to stick the electrode to the skin surface with a non-irritant glue. We have used the same glue as Boter et al. (1966), but we have been able to keep a multipoint electrode glued to a patient for four months, whereas the electrodes of Boter et al., using the glue plus an electrode jelly, often exhibit a skin reaction after 24 hours. This suggests that for long-term use the choice of jelly is important. Kahn (1965) and Boter et al. (1966) have designed ingenious electrodes to overcome the artefacts arising from the relative motion of the electrode and jelly. This problem does not arise with multipoint electrodes since jelly or paste is not used. More recently Fluck and Burgess (1966) have developed press-stud non-irritant jelly chest electrodes for continuous monitoring for periods of a week or more, which they state yield a stable electrocardiogram when the patient is moving.

The present multipoint electrodes are relatively crude and may exhibit initial contact impedances of up to $10 \mathrm{k} \Omega$ at 20 c.p.s. This value of impedance, nevertheless, is satisfactory for use with modern electrocardiographs having input impedances of $1 \mathrm{M} \Omega$ or more, and central terminal averaging resistors substantially higher than $10 \mathrm{k} \Omega$.

The National Aeronautics and Space Administration, Washington D.C. (1966), have now developed a simplified and improved method for recording the electrocardiogram and other biological data in test pilots, which replaces bulky sensors requiring shaving and messy creams. Roman (1966) and Patten, Ramme, and Roman (1966) have perfected high-impedance electrode techniques in which the bare end of a teflon-covered copper wire fourthousandths of an inch in diameter is captured in a conductive fast-drying glue rich in metallic silver which is sprayed on to the chest wall. The electrode area $19 \mathrm{~mm}$. in diameter and $0.5 \mathrm{~mm}$. in thickness is covered by a thin coat of an insulating spray which dries immediately. Application of one electrode, including skin preparation, averages 20 seconds. These electrodes have been used successfully during 700 hours of flight recording and 500 hours of records from working subjects on the ground.

The above reports from The National Aeronautics and Space Administration and evidence to date of the practical value of the multipoint electrode suggests that a dry electrode requiring no skin preparation is likely to replace conventional jelly methods both for telemetry and for routine electrocardiography.

\section{SUMMARY}

An experimental dry multipoint chest electrode requiring no skin preparation with electrode jelly or paste has been used successfully in a preliminary study of patient-monitoring in cardiac infarction by radio-telemetry and for the assessment of cardiac status during physical exercise.

The equipment used and the methods employed are described. The battery-operated British telemetry system, which may be purchased for less than the cost of a modern single-channel electrocardiograph, will permit intermittent monitoring for about 50 hours and, when used continuously, for approximately 10 hours. Batteries which are inexpensive may be quickly replaced.

It is considered that patient-monitoring by telemetry should replace current methods which may increase the emotional tension and anxiety of seriously ill patients already predisposed by cardiac infarction to dangerous cardiac dysrhythmias, and at the same time render emergency treatment at the bedside difficult.

Radio-telemetry is obligatory for the electrocardiographic assessment of cardiac function during physical exercise which precludes the use of conventional electrocardiography, and is of value in helping to establish more valid criteria which will assist the investigation of cardiovascular disease.

We wish to thank Mr. E. P. Childerhouse and Mr. R. S. Mark of Medical and Industrial Equipment Ltd., and Dr. A. Rooker of Aveley Electronics Ltd. for loaning radio-telemetry equipment, and $\mathrm{Mr}$. A. Stevenson of Louis Newmark Ltd. for providing transistorized electrocardiographs for this study. The assistance of the G.P.O. is gratefully acknowledged, and we thank Hewlett-Packard Ltd. and Texas Instruments Ltd., Bedford, who made the electrodes, for their help and co-operation. 


\section{Application of Multipoint Electrodes to Telemetry in Patient-monitoring}

This investigation was supported by research awards from The British Heart Foundation and The NorthWest Metropolitan Regional Hospital Board to one of us (D. L.), who wishes to thank Dr. William Evans for his encouragement and advice in carrying out this research and in the preparation of this paper.

The multipoint electrode is subject to U.K. Patent Applications No. 52253/64 and 44049/65, and in the U.S.A. under the protection of the National Research Development Corporation, Victoria Street, London. R. Ward (Turned Parts) Ltd. of Aberystwyth, and Hewlett-Packard Ltd. in Edinburgh and Palo Alto, California, U.S.A., are carrying out further development of the multipoint electrode which they intend to produce commercially in the near future.

\section{REFERENCES}

Bellet, S., Eliakim, M., Deliyiannis, S., and LaVan, D. (1962) Radioelectrocardiography during exercise in patients with angina pectoris. Circulation, 25, 5 .

Boter, J., den Hertog, A., and Kuiper, J. (1966). Disturbance-free skin electrodes for persons during exercise. Med. biol. Engng., 4, 91.

Ernsting, J. (1966). Multipoint electrocardiography. Lancet, 1, 1429.

Fluck, D. C., and Burgess, P. A. (1966). A press-stud elec- trode for continuous monitoring of the electrocardiogram. Lancet, 1, 1405.

Hill, D. W. (1966). Transmitting physiological signals by telephone. World med. Electron., 4, 108.

Holter, N. J. (1961). New method for heart studies. Science, 134, 1214.

Kahn, A. (1965). Motion artefacts and streaming potentials in relation to biological electrodes. Digest of 6 th. Int. Conf. Med. Electron., Biol. Engng, Tokyo, p. 562.

Keen, H., and Johnson, V. (1966). Multipoint electrocardiography. Lancet, 2, 109.

Lewes, D. (1965). Multipoint electrocardiography without skin preparation. Lancet, $2,17$.

- (1966). Multipoint electrocardiography without skin preparation. World med. Electron., 4, 240.

National Aeronautics and Space Administration, Washington D.C. (1966). Release no. 66-116 (May 17).

Patten, C. W., Ramme, F. B., and Roman, J. (1966). Dry electrodes for physiological monitoring. NASA Technical Note (NASA, TN D-3414).

Robertson, D: (1937). The examination and recording of the human electrocardiogram by means of the cathode-ray oscillograph. F. Instn. elect. Engrs., 81, 497.

Roman, J. (1966). High impedance electrode techniques. Aerospace Med., 37, 790.

Stock, P. (1966). Multipoint electrocardiography. Lancet, $1,1042$.

Turner, R. W. D. (1966). Multipoint electrocardiography. Lancet, 1, 1158.

Wilton-Davies, C. C. (1966). Multipoint electrocardiography. Lancet, 1, 1209. 